\title{
Therapeutics
}

\section{Review: varicella vaccination is effective in children}

\author{
Skull SA, Wang EE. Varicella vaccination - a critical review of the evidence. Arch Dis Child 2001 Aug;85:83-90.
}

\section{QUESTION: What are the benefits and harms of varicella vaccination in children?}

\section{Data sources}

Studies were identified by searching Medline (1966 to 2000) with the terms chickenpox, vaccination, and human, and with methodological terms to retrieve high quality controlled clinical trials and cohort studies; EMBASE/Excerpta Medica; and the Cochrane Library. The internet was searched for position papers and summaries from health organisations and vaccine product information, and reference lists of relevant articles were reviewed.

\section{Study selection}

Studies were selected if they were randomised controlled trials (RCTs) or cohort studies of healthy humans vaccinated with varicella zoster virus (VZV) vaccine and if they assessed the incidence of varicella, zoster, or adverse reactions to vaccination.

\section{Data extraction}

Data were extracted on study design, patient characteristics, length of follow up, vaccine dose, and outcomes (vaccine effectiveness, adverse reactions, transmission of varicella, and risk for herpes).

\section{Main results}

10 reports from 8 RCTs assessed vaccine effectiveness: 2 RCTs of a single dose of VZV showed effectiveness in preventing varicella; 1 showed $100 \%$ protective efficacy at 9 months, and 1 showed $72 \%$ protective efficacy at 29 months. 3 dose-titration RCTs supported the preventive efficacy of VZV. The number needed to vaccinate to prevent 1 additional case of varicella ranged from 6 to 12. One RCT showed no difference in vaccine effectiveness for doses ranging between 439 and 3625 pock-forming units (PFU); a second showed effectiveness at doses $\geqslant 1260$ PFU only; and a third showed effectiveness at doses from 7943 to 10000 PFU. 13 reports from 12 trials assessed adverse reactions; of 3 RCTs of children, none showed an increase in fever or varicella-like rash from VZV over placebo. 1 RCT showed more vaccinerecipients had local reactions than did placebo recipients at 48 hours (27\% v $19 \%)$. Injection-site reactions ranged from $7 \%$ to $30 \%$. Adverse reactions were not related to vaccine dose. Few trials examined transmission of varicella from vaccinated people to others; 1 RCT found seroconversion but no disease in siblings of $\mathrm{VZV}$-vaccinated people. 1 RCT that investigated the risk for herpes zoster in vaccinated people found no zoster in either vaccinated or placebo groups at 9 months.

\section{Conclusion}

Evidence from randomised controlled trials supports the effectiveness of varicella vaccination in children.
Source of funding: not stated.

For correspondence: Dr S Skull, Victorian Infectious Disease. Service, Royal

Melbourne Hospital, Parkville, 3050

Victoria, Australia.

\section{COMMENTARY}

Varicella (chickenpox) is a common contagious disease for which we have had a live attenuated varicella vaccine containing Oka-strain virus for several years. ${ }^{1}$ One of the first target populations was immunocompromised children. Recently, the routine use of varicella vaccine has been considered. The review by Skull and Wang shows, convincingly, that varicella vaccine is effective in preventing varicella. Achievement of herd immunity is difficult, if not impossible, because vaccinated people may develop herpes zoster caused by the wild virus. One obstacle against routine vaccination has been the storage of the vaccines. Earlier varicella vaccine had to be frozen, but a commercial product is now available that can be refrigerated. No commercial product is currently available that can be combined with other routine vaccines.

Insufficient knowledge exists about the occurrence of herpes zoster in vaccinated people as they grow older. However, it seems that zoster is less common among vaccinated people, but it will take several more years before we have evidence of an association.

Vaccination is cost effective for society, but the cost effectiveness is not so obvious to the healthcare organisations that pay the costs of routine childhood vaccinations.. ${ }^{23}$ The review by Skull and Wang shows that varicella vaccine could and should be used more widely than it is. It may be time to implement routine vaccination for all children for varicella.

Matti Uhari, MD, MSc University of Oulu Oulu, Finland

1 Takahashi M, Otsuka T, Okuno Y, et al. Live vaccine used to prevent the spread of varicella in children in hospital. Lancet 1974;2:1288-90.

2 Lieu TA, Cochi SL, Black SB, et al. Cost-effectiveness of a routine varicella vaccination program for US children. JAMA 1994;271:375-81.

3 Diez Domingo J, Ridao M, Latour J, et al. A cost benefit analysis of routine varicella vaccination in Spain. Vaccine 1999;17:1306-11. 